\title{
Localized High-Resolution Proton NMR Spectroscopy Using Stimulated Echoes: Initial Applications to Human Brain in Vivo
}

\author{
J. Frahm, H. Bruhn, M. L. Gyngell, K. D. Merboldt, W. Hänicke, \\ AND R. SAUTER*
}

\begin{abstract}
Max-Planck-Institut für biophysikalische Chemie, Postfach 2841, D-3400 Göttingen, Federal Republic of Germany, and * Bereich Medizinische Technik, Siemens AG, Henkestrasse 27, D-8520 Erlangen, Federal Republic of Germany
\end{abstract}

Received April 22, 1988; revised June 7, 1988

\begin{abstract}
Water-suppressed localized proton NMR spectroscopy using stimulated echoes has been successfully applied to detect metabolites in the human brain in vivo. The STEAM spectroscopy sequence allows single-step localization by exciting three intersecting slices. Water suppression is achieved by preceding chemical-shift-selective (CHESS) rf pulses. High-resolution $(0.05 \mathrm{ppm})$ proton NMR spectra of healthy volunteers have been obtained on a conventional 1.5-T whole-body MRI system (Siemens Magnetom). Volumes-of-interest ( VOI) of $64 \mathrm{ml}\left(4 \times 4 \times 4 \mathrm{~cm}^{3}\right)$ were localized in the occipital area of the brain and spectra were recorded within measuring times ranging from 1 s (single scan) to about $10 \mathrm{~min}$. The experimental procedure is described in detail. Resonance assignments include acetate, $N$-acetyl aspartate, $\gamma$-amino butyrate, glutamine, glutamate, aspartate, creatine and phosphocreatine, choline-containing compounds, taurine, and inositols. Cerebral lactate was found to be at a maximum concentration of $0.5 \mathrm{~m} M$ when assuming $N$-acetyl aspartate in white matter to be $6 \mathrm{~m} M$. (c) 1989 Academic Press, Inc.
\end{abstract}

\section{INTRODUCTION}

Methods, instrumental developments, and medical applications of magnetic resonance imaging (MRI) have matured to a high-quality noninvasive imaging modality that offers excellent soft tissue contrast, high spatial resolution, and measuring times of seconds. However, beyond basic anatomical and functional descriptions such as cine cardiac studies there is still great hope for a biochemical tissue characterization in vivo as, e.g., known from NMR spectroscopy of excised tissues. Although phosphorous NMR spectroscopy enabled new insights into the metabolism of high-energy phosphates in vivo and contributed to the understanding of some muscle diseases (1), its applications often suffer from the limited localization capabilities of surface coils $(2,3)$, topical magnetic resonance $(4)$, or ISIS techniques $(5)$ in combination with short repetition times. Furthermore, even for large volumes-of-interest up to $100 \mathrm{ml}$ the acquisition of a single ISIS-localized phosphorous spectrum typically requires a measuring time of $30 \mathrm{~min}(6)$.

Proton NMR spectroscopy bears the potential of providing access to a much larger number of metabolites. Detailed biochemical information may be obtained not only on the energy metabolism (e.g., lactate production) but also on free amino acids, 
fatty acids, and neurotransmitters. Moreover, with the stimulated echo (STEAM) spectroscopy sequence ( 7 ) a flexible and reliable localization technique that accomplishes both water suppression and spatial localization in a single step with a minimum of only four $90^{\circ} \mathrm{rf}$ pulses is available. This paper describes the implementation of the STEAM spectroscopy method on a conventional 1.5-T MRI system. The experimental procedures for localized high-resolution proton NMR spectroscopy in vivo are outlined in detail, and first applications to normal human brain are reported. A subsequent paper will present relaxation times, concentrations, and regional differences of major cerebral metabolites (8). In a first application to a patient with a meningioma (9) dramatic spectral alterations that correspond to the known biochemistry of this particular tumor have been observed. A more detailed study on the potential of localized proton NMR spectroscopy for tumor differentiation is in preparation (10). Recently, aliphatic proton NMR spectra of human brain in vivo have also been reported by Hanstock et al. (11) using the ISIS technique in combination with surface coils and depth pulses and by Luyten et al. (12) using a modified STEAM sequence.

\section{TECHNICAL ASPECTS}

\section{Stimulated Echo (STEAM) Spectroscopy}

Figure 1 shows a schematic diagram of the stimulated echo radiofrequency ( $\mathrm{rf}$ ) and magnetic field gradient pulse sequence used for water-suppressed localized proton NMR spectroscopy on a standard 1.5-T whole-body MRI system (Siemens Magnetom equipped with an Oxford 2-T Unistat magnet). Three $90^{\circ}$ slice-selection of pulses $(2.56 \mathrm{~ms}$, sinc shape) with a bandwidth of $3400 \mathrm{~Hz}$ resulted in 4-cm slices using $2 \mathrm{~m} \mathrm{~T} \mathrm{~m}^{-1}$ gradients. For a chemical shift range of about $5 \mathrm{ppm}$, i.e., $320 \mathrm{~Hz}$ at $1.5 \mathrm{~T}$, the applied bandwidth corresponds to a chemical-shift uncertainty in the position of the VOI of less than $4 \mathrm{~mm}$, i.e., $\pm 2 \mathrm{~mm}$ for resonance signals in the aliphatic and aromatic regimes, respectively. The bandwidth and the gradient strength were chosen as a practical compromise between positional uncertainties and $\mathrm{rf}$ power deposition caused by short and intense rf pulses with large bandwidths. The position and the dimensions of the VOI may be varied independently for all three axes (i.e.,

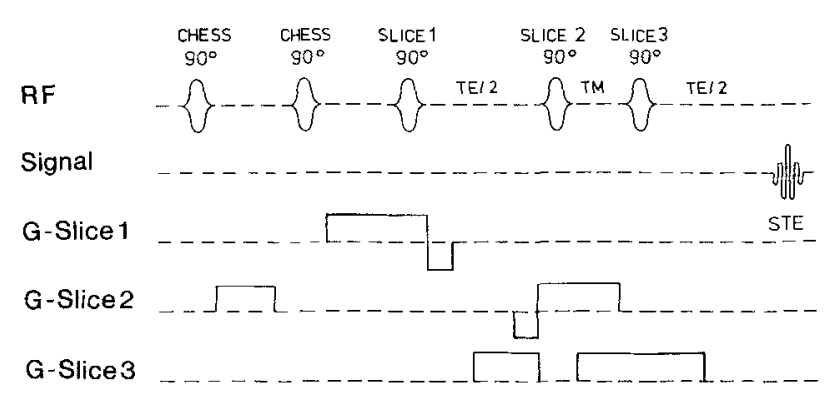

FiG. 1. Schematic diagram of the stimulated echo (STEAM) sequence used for water-suppressed localized proton spectroscopy on a conventional 1.5-T whole-body MRI system. For details see text. 
pulses) allowing flexible adjustments according to anatomical ( 8 ) or pathological requirements $(9,10)$.

The minimum echo times TE and repetition times TR used in the present study were 50 and $1500 \mathrm{~ms}$, respectively. The middle interval was $\mathrm{TM}=30 \mathrm{~ms}$. Acquisition of the second half of the stimulated echo was performed within $1 \mathrm{~s}$ using $2 \mathrm{~K}$ data points and a frequency range of $2 \mathrm{kHz}$. To ensure effective spoiling of the final FID the application of the slice-selection gradient after the third pulse was continued for about $15 \mathrm{~ms}$. No spectral processing of the data has been performed except for zero filling to $4 \mathrm{~K}$ and a mild exponential multiplication of the time-domain data corresponding to a line broadening of $0.2 \mathrm{~Hz}$. Phased absorption spectra are reported directly without baseline correction or resolution enhancement. Both imaging and spectroscopy experiments were carried out using a crossed Helmholtz coil (13) with a diameter of $22 \mathrm{~cm}$.

\section{Magnetic Field Gradient Switches}

The actual gradient waveforms have been carefully optimized with respect to three conditions: (i) generation of a stimulated echo, (ii) dephasing of unwanted echo responses, and (iii) reduction of signal attenuation due to motion and diffusion.

The unambiguous generation of a stimulated echo relies on the presence of dephased transverse magnetizations before application of the second rf pulse. Although Hahn in his original paper about $90^{\circ}-90^{\circ}$ spin echoes and $90^{\circ}-90^{\circ}-90^{\circ}$ stimulated echoes (14) explicitly assumed that the precessing "vector monochromats are allowed to ravel completely in a time $T E / 2 \gg 1 /(\Delta \omega)^{1 / 2}$ before the second pulse is applied" ( $\Delta \omega$, spectral frequency range), this dephasing requirement is not widely recognized. In STEAM imaging (15) dephasing is achieved by application of the read gradient between the first and second rf pulses. In spectroscopy versions dephasing in the first interval may be accomplished by proper switching of the slice-selection gradients as shown in Fig. 1. However, if, e.g., the third slice-selection gradient is refocused after the third if pulse rather than before the second rf pulse, the spectrum obtained from the stimulated echo signal would exhibit a sinusoidal amplitude modulation depending on the echo time TE, on the resonance offsets $\Delta \omega$, and on the phase angle of the rf pulses. Assuming identical rf phases the three-pulse sequence would even resemble a $180^{\circ}-90^{\circ}$ inversion recovery experiment for on-resonance spin moments resulting in a final FID rather than a stimulated echo. In fact, Hahn already stated that the echo signals will be independent of rf phases only as long as the above condition holds $(14)$.

Second, the gradient waveforms selected for STEAM spectroscopy ensure proper dephasing of unwanted echoes by the application of gradients during TM. Figure 2 shows absolute values of the time-domain signals obtained after the third $90^{\circ}$ pulse of a three-pulse sequence in the presence of a constant gradient. The signals in Fig. $2 \mathrm{a}$ refer to the stimulated echo STE at TE/2 as well as to spin echoes generated by the first and second pulses $\mathrm{SE}(1,2)$ at $\mathrm{TE} / 2$ - TM, by the first and third pulses $\operatorname{SE}(1,3)$ at $\mathrm{TE} / 2+\mathrm{TM}$, and by the second and third pulses $\operatorname{SE}(2,3)$ at TM. As shown in Fig. $2 \mathrm{~b}$ a single spoiling gradient during TM eliminates all unwanted echoes. In the spectroscopy sequence shown in Fig. 1 spoiling in the middle interval is 


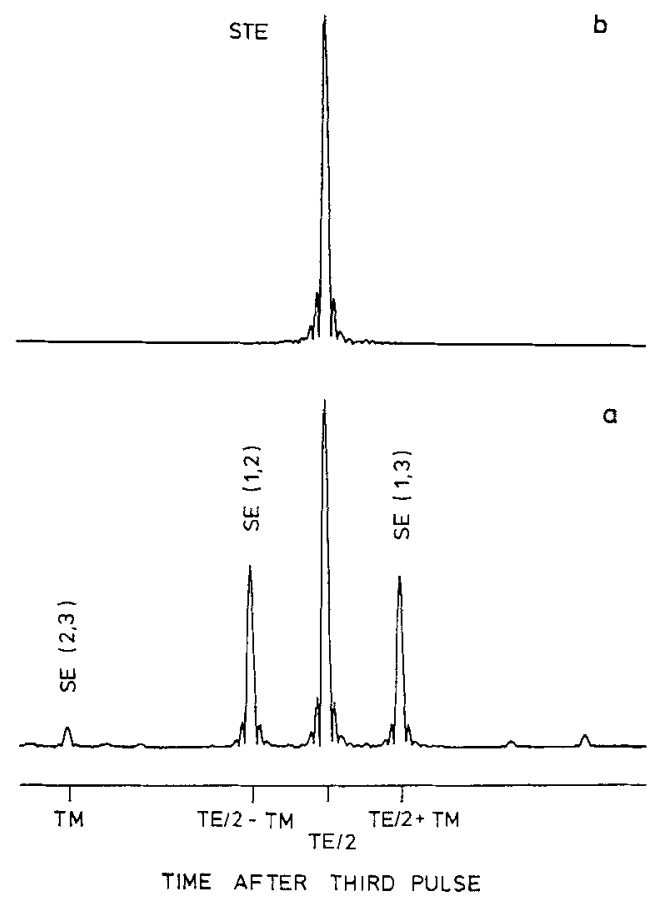

FIG. 2. ( $\mathrm{a}$ and $\mathrm{b}$ ) Sixty-four-megahertz (1.5-T) proton time-domain MR signals of a stimulated echo rf pulse sequence as shown in Fig. 1. Signals are displayed as a function of time after the final $90^{\circ}$ pulse and represent absolute intensities of echoes which have been recorded in the presence of a constant gradient applied throughout the entire sequence (a) in the absence and (b) in the presence of an additional spoiler gradient pulse in the interval TM. Echo assignments refer to STE, stimulated echo; SE $(1,2)$, spin echo of the first and second pulses; $\operatorname{SE}(1,3)$, spin echo of the first and third pulses; $\operatorname{SE}(2,3)$, spin echo of the second and third pulses. Further residual signals in (a) are caused by transmitter offsets of the preceding CHESS pulses.

achieved by the slice selection gradients associated with the second and third rf pulses. Of course, net spoiling only results from asymmetric gradient switches that avoid the generation of new gradient echoes at the end of the middle interval or after termination of the gradient in the third interval, respectively.

Finally, the gradient waveforms of Fig. 1 are optimized to reduce signal attenuation by unwanted "phase" encoding of moving spins. Motion in the presence of improper gradient switches has been demonstrated to result in considerable signal loss (16). Even capillary flow causes problems at long echo times. Although signals may be regained using motion-compensating gradients as commonly employed in MRI (17) this approach normally requires strong gradients which may lead to diffusion attenuation. In the present brain studies phase errors due to motion have been minimized by shortening the timings between rephasing and dephasing lobes of the slice-selection gradients. In particular, the time between the first and the final application of the third slice-selection gradient (G-Slice 3 in Fig. 1) is kept constant to $55 \mathrm{~ms}$ also for different echo times. Thus, an interference of motion and diffusion with $T_{2}$ determinations is avoided. 


\section{RESULTS AND DISCUSSION}

A typical protocol for the investigation of normal volunteers included (i) FLASH MRI in all three orientations to adequately determine the VOI, (ii) global shimming of the head, (iii) localized shimming on the selected VOI, (iv) localized optimization of the $90^{\circ}$ slice-selection if pulses, ( $v$ ) localized optimization of water suppression, and (vi) proton spectroscopy using differing echo times and repetition times. Total investigational times were of the order of $1.5 \mathrm{~h}$.

\section{MR Imaging of the VOI}

The localization achieved with the STEAM sequence may be easily verified by imaging of the VOI. For this purpose the sequence in Fig. 1 has been used without CHESS pulses. A phase-encoding gradient and a reversed read gradient were added after the final $90^{\circ} \mathrm{rf}$ pulse yielding an echo signal at $\mathrm{TE}=30 \mathrm{~ms}$. To avoid unnecessarily large partial volume effects in the third dimension the $4-\mathrm{cm}^{3}$ VOI was covered by three $1-\mathrm{cm}$-thick slices $0.5 \mathrm{~cm}$ apart from each other. Simultaneous localized multislice STEAM imaging ( 15 ) was performed by using triple application of the final $90^{\circ}$ rf pulse. While the echo time remains constant, the duration of the middle increased according to 10,35 , and $60 \mathrm{~ms}$. An example is given in Fig. $3 \mathrm{~b}$ where the VOI has been positioned in the frontal part of the ventricles to facilitate comparisons with conventional MR images. Figure 3a is a standard FLASH image (18-21) which was normally employed for rapid selection of the VOI. The images in Figs. $3 \mathrm{c}$ and $3 \mathrm{~d}$ refer to magnified views of the desired section in Fig. 3a (software zoom) and of the experimentally selected section in Fig. 3b (STEAM localization). Obviously, there is perfect agreement, and to minimize investigational times only FLASH MRI was performed in subsequent volunteer and patient studies.

\section{Magnetic Field Homogeneity}

Shimming, and in particular localized shimming, is of extreme importance for performing high-quality spectroscopy experiments in vivo. Magnetic field inhomogeneities sacrifice both spectral resolution and signal-to-noise in reducing peak intensities of broadened lines. Localized shimming (without water suppression) is another advantage of STEAM spectroscopy sequences. It turned out that water proton linewidths (at half-height) of $2 \mathrm{~Hz}$ could be achieved in a $100 \mathrm{~m} M$ lactic acid phantom using a 64-ml VOI in the center of the 1.5-T magnet. Two well-separated resonance lines were observed that correspond to the $7-\mathrm{Hz} J$ splitting of the lactate methyl protons.

For in vivo brain studies, the shimming procedure started with a global FID shim of the entire head. Typically, water proton linewidths of $15-20 \mathrm{~Hz}$ were obtained within $5 \mathrm{~min}$ corresponding to about $0.25 \mathrm{ppm}$ at $1.5 \mathrm{~T}$. It should be emphasized that the resonance signal of the entire head represents a spatially heterogeneous distribution of individual resonances with different linewidths. Therefore, the linewidth for a localized VOI strongly depends on the actual position of the VOI within the head and is not necessarily given by the "global" value. For example, for a VOI localized in the midbrain the local linewidth was found to be smaller than the global value by 

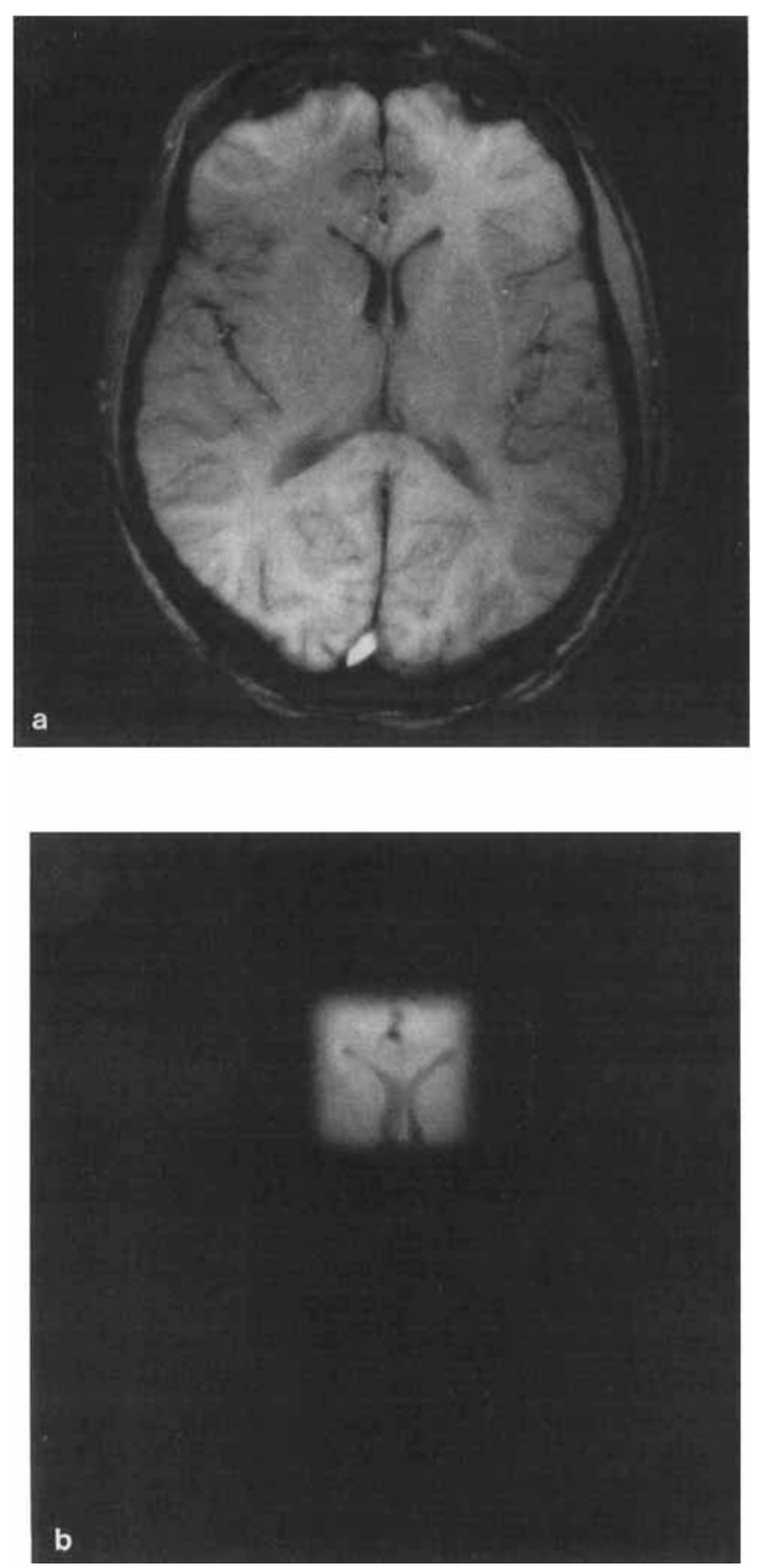

FIG. 3. (a-d) Sixty-four-megahertz (1.5-T) proton MR images of the brain of a normal volunteer. (a) Axial FLASH image; (b) localized VOI $\left(4 \times 4 \times 4 \mathrm{~cm}^{3}\right)$ STEAM image; (c) zoomed section of the FLASH image corresponding to the desired VOI; (d) magnification of the VOI STEAM image by the same factor as used for $(c)$. 

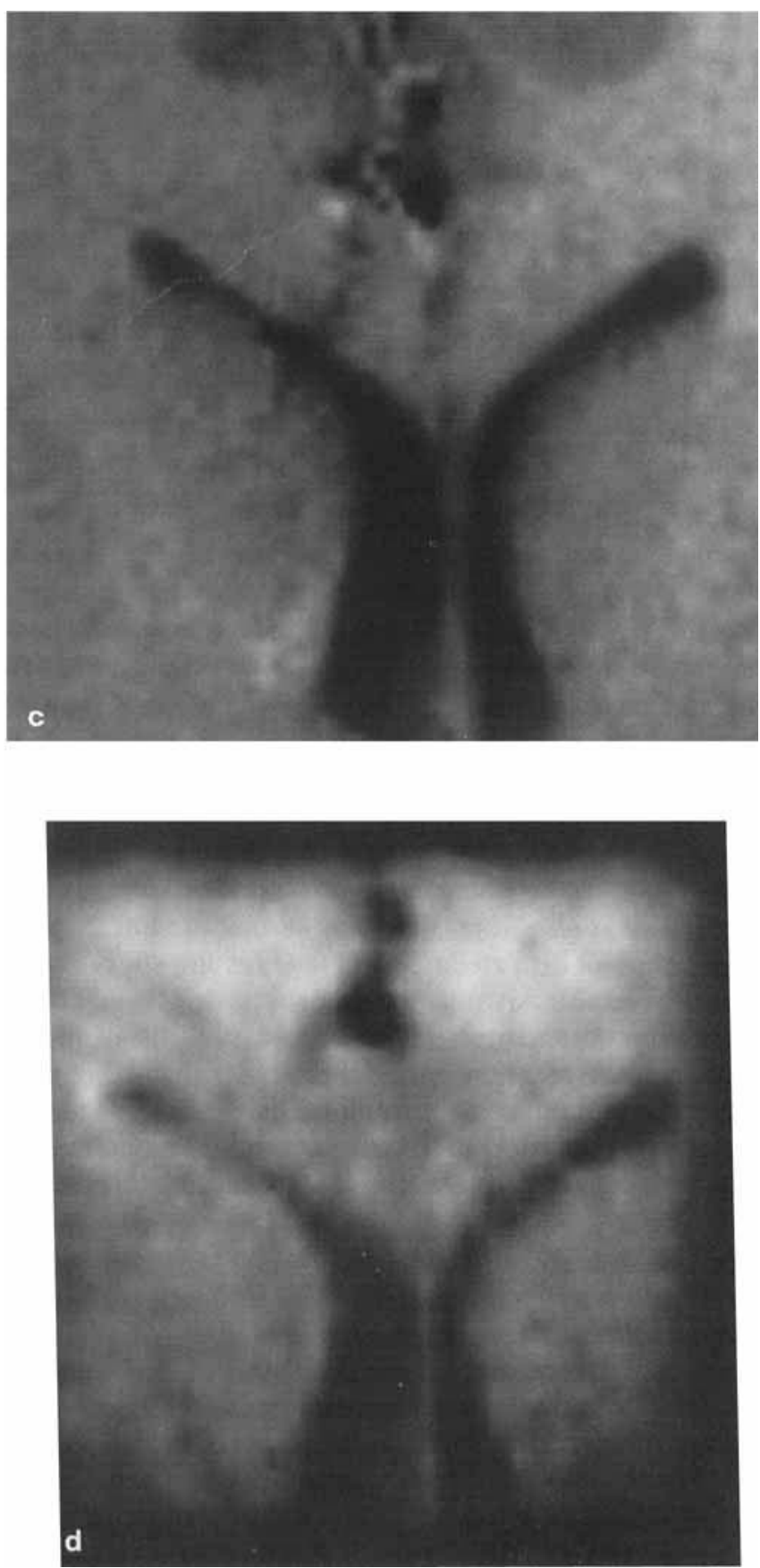

FIG. 3-Continued. 
about a factor of 2 using the same shim values. On the other hand, for a VOI localized in the frontal brain in a position of known susceptibility differences the linewidth was even worse by a factor of 2 corresponding to the broad resonances contributing to the "foot" of the global resonance line.

Nevertheless, in our studies it was always possible to improve the homogeneity in arbitrary positions in the brain to spectral linewidths of 4-6 Hz. Typically, localized shimming at $\mathrm{TE}=50 \mathrm{~ms}$ was performed for about $10-15 \mathrm{~min}$ using only linear gradients. Similar results were obtained on patients including a variety of brain tumors $(9,10)$ and are in agreement with results reported by others; see, e.g., $(22,23)$. The fact that only linear shim gradients are required to compensate for susceptibilityinduced local gradients is also in agreement with the ability to compensate susceptibility artifacts in FLASH images by readjustments of the (linear) slice-selection gradient (24). On the contrary, artificially introduced gradients in the vicinity of metallic surgical clips turned out to be highly nonlinear in character (25).

\section{Water Suppression}

Water suppression in a STEAM spectroscopy sequence is most easily performed by preceding chemical-shift-selective (CHESS) excitation pulses (26) applied to the unwanted resonance frequency. The excited transverse magnetizations are dephased by subsequent gradients prior to the localization sequence (compare Fig. 1). Here two Gaussian-shaped CHESS pulses have been employed with an intermediate spoiler gradient of $50 \mathrm{~ms}$ duration. The first slice-selection gradient was applied $15 \mathrm{~ms}$ before the corresponding if pulse. Since the CHESS pulses only affect spins within a narrow range of frequencies around the water signal the resulting spectrum is not contaminated in other regions. It is also important to note that the lineshape of the residual water resonance is not at all distorted due to the Gaussian suppression pulses. Both features represent considerable advantages compared to binomial or DANTE rf pulse sequences often used for water suppression. In fact, such frequency-selective pulse trains have been introduced as improvised solutions for high-resolution NMR spectrometers not equipped with pulse shape modulators. Since these pulse trains possess a periodic excitation spectrum they may cause amplitude modulations in frequency regimes of interest and, therefore, may complicate spectral interpretations and quantitative evaluations.

In the STEAM spectroscopy sequence water suppression is required only within the VOI. The optimization was performed after shimming by adjusting the flip angles of the CHESS pulses such that the residual water proton signal is minimized. In addition to $T_{2}$ attenuation of the water resonance CHESS pulses with a duration of 25.6 ms and a bandwidth of $60 \mathrm{~Hz}$ yielded suppression factors of 35-60 in single-scan fully relaxed spectra at TE $=50 \mathrm{~ms}$. Total water suppression includes differential $T_{1}$ (saturation) and $T_{2}$ (attenuation) effects. Typically, signal ratios of 40 and 10 were found for residual water and $N$-acetyl aspartate at TE $=50$ and $270 \mathrm{~ms}$, respectively.

\section{Spectroscopic Investigations}

Figure 4 depicts cross-sectional FLASH MR images of a normal volunteer defining the VOI selected for localized proton NMR spectroscopy by $4 \times 4-\mathrm{cm}^{2}$ squares. The 
VOI has been located within the occipital area of the brain and predominantly contains white matter. The excellent signal-to-noise ratio of the spectroscopic investigations may be judged from the single-scan water-suppressed localized proton NMR spectrum shown in Fig. 5a. The echo time was $\mathrm{TE}=50 \mathrm{~ms}$ and the VOI had a size of $64 \mathrm{ml}$ as indicated in Fig. 4. Resonance assignments were made to major metabolites such as $N$-acetyl aspartate (NAA), creatine ( $\mathrm{Cr}$ ) and phosphocreatine ( $\mathrm{PCr}$ ), and choline-containing compounds (Cho). More detailed studies obviously require multiple acquisitions as demonstrated in Fig. $5 \mathrm{~b}$ for a fully relaxed spectrum obtained from eight scans with a repetition time of TR $=6000 \mathrm{~ms}$. By the recording of spectra at differing TR values the $T_{1}$ relaxation times of most cerebral metabolites were found to be less than $1500 \mathrm{~ms}(8)$. Thus, the signal-to-noise per unit time may be markedly improved by using repetition times of the order of $T_{1}$.

In order to allow resonance assignments without $T_{1}$ saturation effects Fig. 6 again shows the aliphatic resonances of a fully relaxed localized proton NMR spectrum obtained at $\mathrm{TE}=50 \mathrm{~ms}$ using 128 scans and $\mathrm{TR}=6000 \mathrm{~ms}$. Resonance frequencies are referenced to the strong singlet methyl group from NAA at $2.0 \mathrm{ppm}$. The assignments are summarized in Table 1 including information from spectra recorded at $\mathrm{TR}=1500$ ( 512 scans) and differing echo times. Chemical shifts are given with an accuracy of $0.05 \mathrm{ppm}$, while chemical-shift differences were accurate to $1-2 \mathrm{~Hz}$. Metabolites detected include methylene protons from acylglycerides, acetate, $\mathrm{N}$-acetyl aspartate, $\gamma$-aminobutyric acid, glutamate, glutamine, aspartate, creatine and phosphocreatine, choline-containing compounds such as phosphocholine and glycerophosphocholine, taurine, and inositols.

Although in accordance with spectroscopic findings from extracts (27-31) and our own high-resolution studies on pertinent amino acids in solution, the resonance assignments of glutamine, GABA, aspartate, taurine, and inositol have mainly been confirmed by applying the same localization sequence as that used for in vivo studies (same MRI system, same volume, similar TR and TE values, same investigational protocol) to sufficiently large phantoms comprising aqueous solutions $(\mathrm{pH} \approx 7)$ of the respective compounds. These experiments turned out to be extremely important as many spectral features observed with conventional high-field NMR spectrometers undergo significant changes at lower field strengths and for the echo times employed in vivo. In particular, many proton spectra are no longer first-order spectra at $1.5 \mathrm{~T}$ where the typical $J$ splitting of $5-10 \mathrm{~Hz}$ corresponds to $0.1-0.15 \mathrm{ppm}$, and, therefore, often approaches the chemical-shift difference of coupled spins. Complex lineshapes that become even more complicated by echo modulation effects and potential overlap with other multiplets may result. A typical example is taurine. High-resolution spectra exhibit two multiplets for the $\mathrm{N}-\mathrm{CH}_{2}$ and $\mathrm{S}_{-} \mathrm{CH}_{2}$ groups at 3.2 and $3.4 \mathrm{ppm}$, respectively. However, under the present experimental conditions, these two multiplets collapse into a broad resonance peaked at $3.3 \mathrm{ppm}$. Another interesting case is inositol. From the variety of signals present in the 3-4 ppm range of high-field spectra, only a single sharp resonance line at $3.5 \mathrm{ppm}$ remains at $\mathrm{TE}=50 \mathrm{~ms}$ which may be attributed to the $\mathrm{CH}$ ring protons in 4- and 6-position. At $\mathrm{TE}=270 \mathrm{~ms}$ almost no signal is retained. Since this finding is in agreement with the in vivo spectra the 3.5 ppm resonance signal has been assigned to inositol. $\mathrm{The} \mathrm{CH}_{2}$ singlet resonance signal from glycine appears at the same position but has a considerably longer $T_{2}$ relaxation 
FRAHM ET AL.
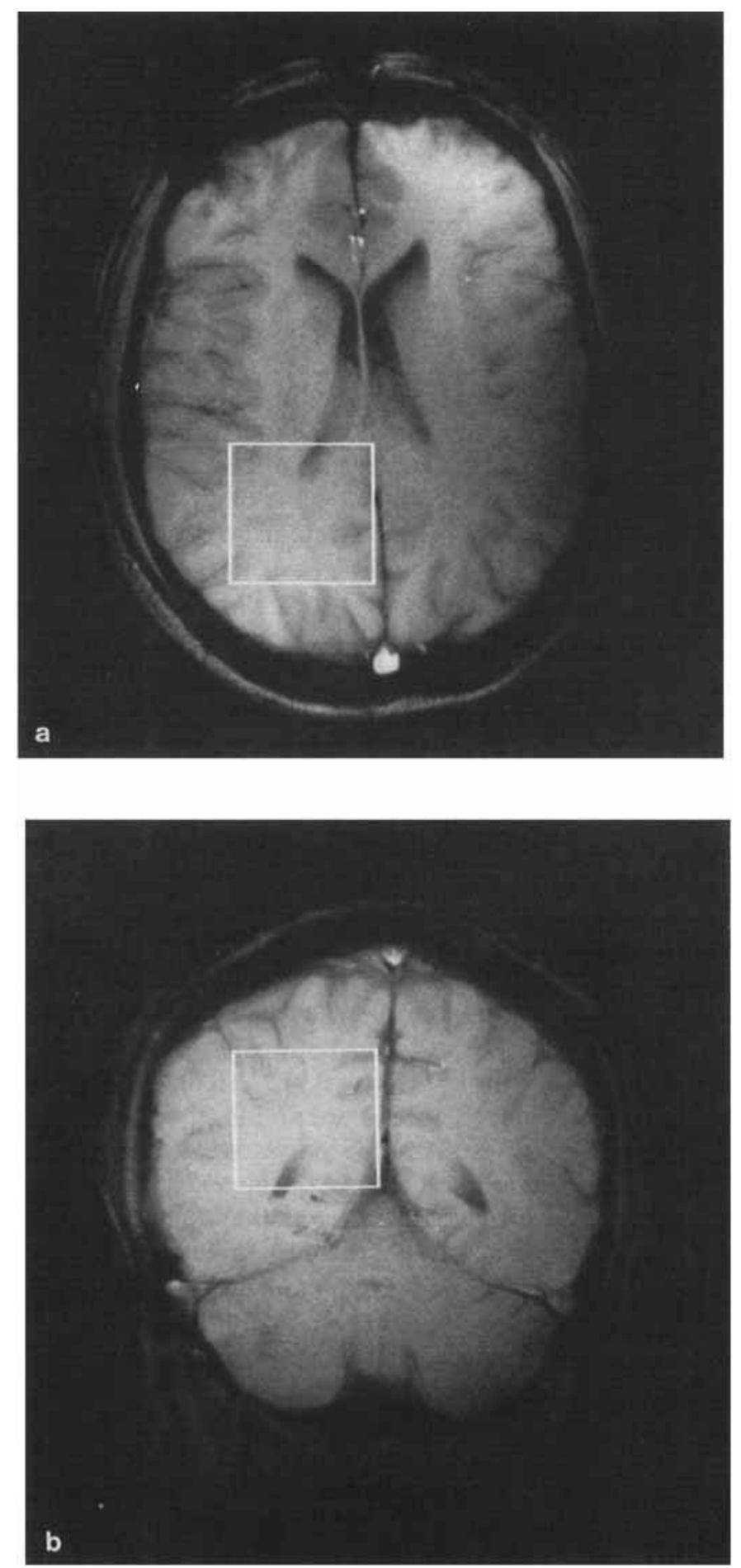


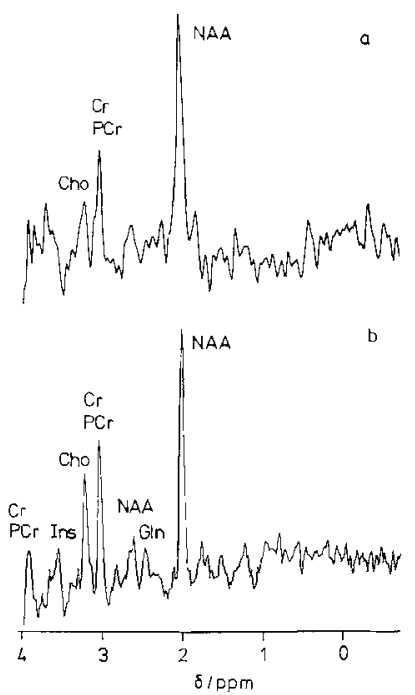

FIG. 5. ( $\mathrm{a}$ and $\mathrm{b}$ ) Sixty-four-megahertz (1.5-T) water-suppressed localized proton MR spectra of a normal volunteer ( $64 \mathrm{ml}$ VOI in the occipital area as indicated in Fig. 4). Fully relaxed spectra at $\mathrm{TE}=50 \mathrm{~ms}$ ( $\mathrm{TM}=30 \mathrm{~ms}$ ). (a) Single scan; (b) eight scans, $\mathrm{TR}=6000 \mathrm{~ms}$. Resonance assignments are made to acetate (Ac), $N$-acetyl aspartate (NAA), glutamine $(\mathrm{Gln})$, creatine $(\mathrm{Cr})$ and phosphocreatine $(\mathrm{PCr})$, cholinecontaining compounds (Cho), and inositol phosphates (Ins).

time. Residual signal contributions in the $\mathrm{TE}=270 \mathrm{~ms}$ in vivo spectra may be due to small contributions from glycine. Further residual signals with large linewidths and/or complex multiplet structures are observed in the spectral ranges 7.0-7.5 and $8.0-8.5 \mathrm{ppm}$. They may be due to exchangeable amide protons of cytosolic proteins and aromatic protons in adenine nucleotides, respectively. In most cases the detection of aromatic resonances in proton NMR studies in vivo is complicated by their short $T_{2}$ relaxation times yielding strong signals only at very short echo times. Resonances from histidine protons at $\mathrm{TE}=20 \mathrm{~ms}$ have been reported by Gadian et al. (32). Strong aromatic resonances from adenine nucleotides have been observed in certain types of tumors $(10)$.

In general, even small spectral details were perfectly reproducible in different investigations and with different volunteers. This indicates that there is negligible human diversity for the steady-state concentrations of these cerebral metabolites. The small and slightly variable lipid signals may be explained by small amounts of free fatty acids present in brain tissue or even by some triglycerides associated with vascular structures present in the VOI. In fact, the "average" lipid concentration of approximately $1 \mathrm{~m} M$ estimated from the in vivo spectra of a 64-mI VOI translates into a

FIG. 4. ( $a$ and b) Sixty-four-megahertz (1.5-T) axial (a) and coronal (b) FLASH MR images of a normal volunteer defining the positions and dimensions of the VOI in the occipital area of the brain selected for localized spectroscopy. The size of the VOI is $4 \times 4 \times 4 \mathrm{~cm}$ corresponding to a volume of $64 \mathrm{ml}$. Signal homogeneity of the images is slightly degraded due to the rf homogeneity profile of the used coil. 


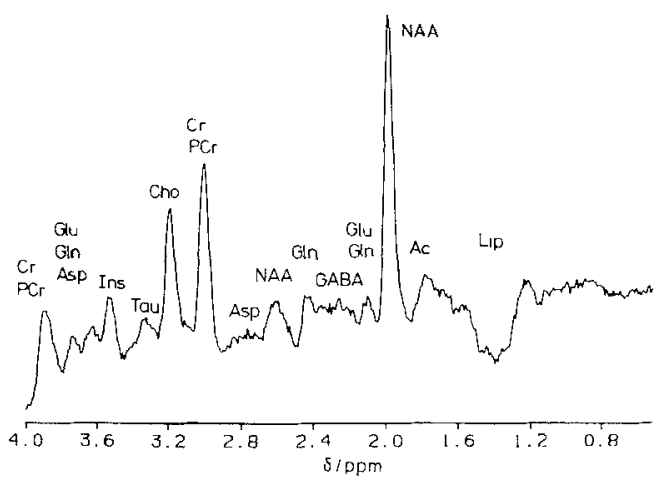

FIG. 6. Sixty-four-megahertz (1.5-T) water-suppressed localized proton MR spectrum of a normal volunteer $(64 \mathrm{ml}$ VOI in the occipital area as indicated in Fig. 4) at $\mathrm{TE}=50 \mathrm{~ms}(\mathrm{TM}=30 \mathrm{~ms})$ using 128 scans and TR $=6000 \mathrm{~ms}$. Resonance assignments are made to lipids (Lip), acetate (Ac), $N$-acetyl aspartate (NAA), $\gamma$-aminobutyric acid (GABA), glutamine (Gln), glutamate (Glu), aspartate (Asp), creatine (Cr) and phosphocreatine ( $\mathrm{PCr}$ ), choline-containing compounds ( $\mathrm{Cho})$, taurine ( $\mathrm{Tau}$ ), and inositol phosphates (Ins). For details see text.

"fat" partial volume of the VOI of only $64 / 60000 \mathrm{ml}$ or about $1 \mu$ lif one assumes a $60 \mathrm{M}$ lipid methylene proton concentration as in marrow or adipose tissue.

A further remark must be made with respect to the detectability and quantification of metabolites in proton NMR spectra. It is well known that dynamical properties such as short $T_{2}$ values for tightly bound molecules or the participation of a molecular group in rapid proton exchange play an important role for the spectral appearance of a certain proton. Further complications may arise from $J$ splitting, strong coupling, $J$ modulation, and overlap of multiplets. A typical example in vivo is given by the surprisingly small signals obtained from glutamate which is known to be present in concentrations of the order of $10 \mathrm{~m} M$. A thorough biochemical correlation of NMR results therefore requires detailed knowledge about the solution proton spectrum of a metabolite at the same field strength as well as about its dynamical properties as expressed by the $T_{1}$ and $T_{2}$ relaxation times of the individual resonance signals.

The influence of strong $T_{2}$ attenuations is demonstrated in Fig. 7 for the aliphatic resonances of a localized proton NMR spectrum in vivo at $\mathrm{TE}=270 \mathrm{~ms}$ using 512 scans and TR $=1500 \mathrm{~ms}$. Assignments are as above and mainly refer to small mobile metabolites with singlet and/or methyl proton resonances with relatively long $T_{2}$ relaxation times. A small but reproducible resonance from the lactate methyl group at $1.3 \mathrm{ppm}$ is borne out by the decay of overlapping lipid resonances with much shorter $T_{2}$ relaxation times. At TE $=270 \mathrm{~ms} J$ modulation effects on the lactate methyl protons $(J=7 \mathrm{~Hz})$ are excluded. Based on a concentration of $6 \mathrm{~m} M$ for $N$ acetyl aspartate in human brain $(33,34)$ and assuming similar $T_{1}$ and $T_{2}$ relaxation times for the methyl protons in lactate and NAA, the concentration of cerebral lactate is estimated to a maximum of $0.5 \mathrm{~m} M$. This value is by a factor of 4 lower than that determined from rat or mouse brain rapidly frozen in situ; see, e.g., (35). It is in agreement with recent findings of others $(11,12)$. The ability of detecting cerebral lactate using localized proton NMR spectroscopy is of great biochemical and medical 
TABLE 1

Tentatively Assigned and Unassigned Resonances of Cerebral Metabolites Detected in Localized Proton NMR Spectra of Human Brain in Vivo (Predominantly White Matter)

\begin{tabular}{llll}
\hline \multicolumn{1}{c}{ Metabolite } & Abbr. & Molecular group & Chemical shift \\
\hline Lipids & Lip & $\mathrm{CH}_{3}, \mathrm{CH}_{2}$ & $0-2$ \\
Lactate & Lac & $\mathrm{CH}_{3}$ & 1.3 \\
Acetate & $\mathrm{Ac}$ & $\mathrm{CH}_{3}$ & 1.85 \\
$\gamma$-Amino butyrate & $\mathrm{GABA}$ & $\gamma-\mathrm{CH}_{2}$ & 2.25 \\
$N$-Acetyl aspartate & $\mathrm{NAA}$ & $\mathrm{CH}_{3}$ & 2.0 \\
& & $\beta-\mathrm{CH}_{2}$ & 2.6 \\
Glutamate & $\mathrm{Glu}$ & $\beta-\mathrm{CH}_{2}$ & 2.1 \\
& & $\gamma-\mathrm{CH}_{2}$ & 2.35 \\
& & $\alpha-\mathrm{CH}_{2}$ & 3.75 \\
Glutamine & $\mathrm{Gln}$ & $\beta-\mathrm{CH}_{2}$ & 2.1 \\
& & $\gamma-\mathrm{CH}_{2}$ & 2.45 \\
& & $\alpha-\mathrm{CH}_{2}$ & 3.75 \\
Aspartate & $\mathrm{Asp}$ & $\beta-\mathrm{CH}_{2}$ & 2.8 \\
Creatine & $\mathrm{Cr}$ & $\mathrm{N}-\mathrm{CH}_{3}$ & 3.0 \\
& & $\mathrm{~N}-\mathrm{CH}_{2}$ & 3.9 \\
Phosphocreatine & $\mathrm{PCr}$ & $\mathrm{N}-\mathrm{CH}_{3}$ & 3.0 \\
& & $\mathrm{~N}-\mathrm{CH}_{2}$ & 3.9 \\
Cholines & $\mathrm{N}-\left(\mathrm{CH}_{3}\right)_{3}$ & 3.2 \\
Taurine & $\mathrm{Cho}$ & $\mathrm{N}-\mathrm{CH}_{2}, \mathrm{~S}-\mathrm{CH}_{2}$ & 3.3 \\
Inositols & $\mathrm{Tau}$ & $\mathrm{H} 4, \mathrm{H}_{6}$ & 3.5 \\
Glycine & $\mathrm{Ins}$ & $\mathrm{CH}$ & 3.5 \\
Unidentified & $\mathrm{Gly}$ & & $7-7.5$ \\
Unidentified & & & $8-8.5$ \\
\hline
\end{tabular}

Note. Chemical shifts are given in parts per million relative to NAA (2.00 ppm).

importance. Markedly increased lactate levels have been observed in certain brain tumors and areas of cerebral infarction using STEAM spectroscopy (10).

\section{CONCLUSIONS}

The present study demonstrates the feasibility of proton NMR spectroscopy in vivo on a conventional 1.5-T MRI system. It clearly indicates that high-resolution NMR studies in man may be performed on a routine basis offering new biochemical insights in vivo as well as clinical applications to various states of metabolic or biochemical disorders $(9,10)$. In particular, proton NMR spectra provide access to the pool of free amino acids (glutamate, glutamine, taurine, aspartate, NAA), to glycolysis (lactate), to the tricarboxylic acid cycle (glutamate), to the metabolism of neurotransmitters (GABA, glutamate), and possibly to intracellular signal transduction (inositols; see, e.g., (36)).

The ability of the STEAM spectroscopy sequence to reliably localize proton NMR signals in an image-selected VOI is considered to be a distinct advantage compared to alternative approaches. The signal-to-noise ratios obtained for a $64-\mathrm{ml}$ VOI and 


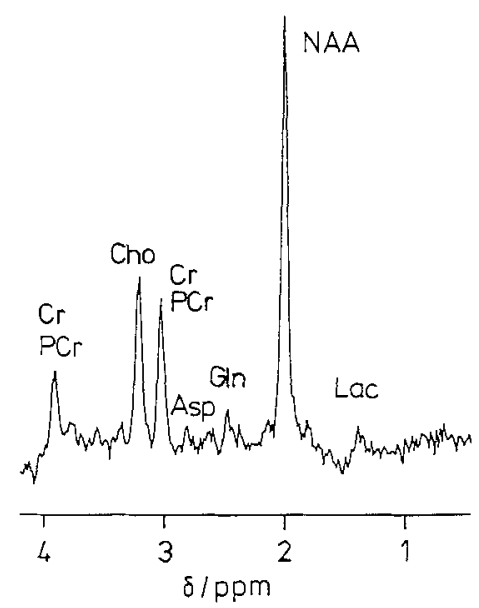

FIG. 7. Sixty-four-megahertz (1.5-T) water-suppressed localized proton MR spectrum of a normal volunteer $(64 \mathrm{ml}$ VOI in the occipital area as indicated in Fig. 4) at TE $=270 \mathrm{~ms}(\mathrm{TM}=30 \mathrm{~ms})$ using 512 scans and $\mathrm{TR}=1500 \mathrm{~ms}$. Resonance assignments are made to lactate (Lac), $N$-acetyl aspartate (NAA), glutamine (GIn), aspartate (Asp), creatine $(\mathrm{Cr})$ and phosphocreatine $(\mathrm{PCr})$, and choline-containing compounds (Cho).

for measuring times of up to 10 min are extremely promising. In future applications the signal-to-noise ratio may be further improved by factors of about 3-4 by simple modifications of the equipment such as smaller coils with better filling factors or preamplifiers optimized for the amplification of small spectroscopic signals rather than for MRI. This will allow a reduction of either the measuring time or the VOI size. In addition, shorter echo times of the order of 10-15 ms may be used to enhance signal contributions from resonances with short $T_{2}$ relaxation times as well as facilitate applications to liver and cardiac spectroscopy.

Proton NMR linewidths of 4-6 Hz have been routinely achieved after localized shimming in all parts of the brain using only linear gradients. This finding proves that the importance of local field gradients induced by biological susceptibility differences has been overestimated in the past. Previous in situ experiments such as, e.g., surface coil brain studies of laboratory animals with the scalp removed might have been complicated by strong susceptibility gradients artificially created at the air-tissue interfaces.

\section{ACKNOWLEDGMENT}

Financial support by the Bundesminister für Forschung und Technologie (BMFT) of the Federal Republic of Germany (Grant 01 VF 8606/6) is gratefully acknowledged.

\section{REFERENCES}

1. G. K. RADDA, Science 233, 640 (1986).

2. J. J. H. Ackerman, T. H. Grove, G. G. Wong, D. G. Gadian, and G. K. Radda, Nature (London) 283, 167 (1980).

3. A. HAASE, W. HäNICKE, AND J. FRAHM, J. Magn. Reson. 56, 401 (1984). 
4. R. E. Gordon, P. E. HANLEy, D. SHaW, et al., Nature (London) 287, 736 (1980); Prog. NMR Spectrosc. 15, 1 (1982).

5. R. J. ORDidGe, A. CONNElly, AND J. A. B. LohmAN, J. Magn. Reson. 66, 283 (1986).

6. C. M. Segebarth, D. F. Baleriaux, D. L. Arnold, P. R. Luyten, and J. A. den Hollander, Radiology 165, 215 (1987)

7. J. Frahm, K. D. Merboldt, and W. HÄnicke, J. Magn. Reson. 72, 502 (1987); German Patent Offenlegungsschrift P 3445689.9 (Priority: December 14th, 1984).

8. J. Frahm, H. Bruhn, M. L. Gyngell, K. D. Merboldt, W. Hänicke, and R. SAuter, submitted for publication.

9. J. Frahm, H. Bruhn, M. L. GYNGell, K. D. MerboldT, W. HäniCKE, AND R. SAuter, submitted for publication.

10. H. Bruhn, J. Frahm, M. L. Gyngell, K. D. Merboldt, W. Hänicke, R. Sauter, and C. HamBURGER, submitted for publication.

11. C. C. Hanstock, D. L. Rothman, T. Jue, and R. G. Shulman, J. Magn. Reson. 77, 583 (1988); C. C. Hanstock, D. L. Rothman, J. W. Pritchard, T. Jue, and R. G. Shulman, Proc. Natl. Acad. Sci. USA 85, 1821 (1988).

12. P. R. Luyten, C. Segebarth, D. Baleriaux, and J. A. Den Hollander, Radiology P 165, 66 (1987).

13. H. REQUARDT, J. OFFERMANN, AND R. SAUTER, submitted for publication.

14. E. L. Hahn, Phys. Rev. 80, 580 (1950).

15. J. FRAHM, K. D. MERBoldT, W. HÄNICKe, AND A. HAASE, J. Magn. Reson. 64, 81 (1985).

16. M. L. Gyngell, J. Frahm, K. D. Merboldt, W. Hänicke, and H. Bruhn, J. Magn. Reson. 77, 596 (1988).

17. P. M. Pattany, J. J. Phillips, L. C. Chiu, J. D. Lipcamon, J. L. Duerk, J. M. MCNally, and S. N. Mohapatra, J. Comput. Assist. Tomogr. 11, 369 (1987).

18. A. HaAse, J. Frahm, D. Matthaei, W. Hänicke, and K. D. Merboldt, J. Magn. Reson. 67, 258 (1986).

19. J. Frahm, A. HaASE, AND D. MatThaeI, Magn. Reson. Med. 3, 321 (1986).

20. J. FRAHM, K. D. MERBOLDT, AND W. HÄNICKE, J. Magn. Reson. 72, 307 (1987).

21. J. FRAHM, Naturwissenschaften 74, 415 (1987).

22. I. J. Cox, G. M. Bydder, D. G. Gadian, I. R. Young, E. Proctor, S. R. Williams, and I. Hart, J. Magn. Reson. 70, 163 (1986).

23. I. J. CoX, D. J. Bryant, B. D. Ross, I. R. YounG, D. G. Gadian, G. M. Bydder, S. R. Williams, A. L. Busza, AND T. E. BATES, Magn. Reson. Med. 5, 186 (1987).

24. J. Frahm, K. D. Merboldt, AND W. Hänicke, Magn. Reson. Med. 6, 474 (1988).

25. J. Frahm, W. Hänicke, K. D. Merboldt, and R. MCKinneY, JR., Technical Report No. 11/ 0000/1 19, Edward Weck and Company, Inc. Research Triangle Park, NC, February 1988.

26. A. HaAse, J. Frahm, W. Hänicke, and D. Matthael, Phys. Med. Biol. 30, 341 (1985).

27. C. Arus, Y. C. Chang, AND M. Barany, Physiol. Chem. Phys. Med. NMR 17, 23 (1985).

28. S. Cerdan, R. PARrilla, J. SANTORO, AND M. Rico, FEBS Lett. 187, 167 (1985).

29. G. W. MAYR AND W. DIETRICH, FEBS Lett. 213, 278 (1987).

30. S. R. Williams and D. G. Gadian, Q.J. Exp. Physiol. 71, 335 (1986).

31. O. A. C. Petroff, E. Hogan, J. Johansen, and A. L. Kleinhaus, Comp. Biochem. Physiol. B 87, 927 (1987).

32. D. G. Gadian, E. Proctor, S. R. Williams, E. B. Cady, and R. M. Gardiner, Magn. Reson. Med. 3, 150 (1986).

33. H. H. Tallan, in "Free Amino Acid Pools" (J. T. Holden, Ed.), pp. 471-485, Elsevier, Amsterdam, 1962.

34. M. C. Fleming AND O. H. Lowry, J. Neurochem. 13, 779 (1966).

35. H. MCILWAIN AND H. S. BAChELARD, "Biochemistry and the Central Nervous System," 5th ed., p. 90, Churchill Livingston, Edinburgh, 1985.

36. M. J. BERRIDGE AND R. F. IRVINE, Nature (London) 312, 315 (1984). 